\title{
Magnetic Resonance Imaging-Detected Intracranial Extension in the T4 Classification Nasopharyngeal Carcinoma with Intensity-Modulated Radiotherapy
}

\author{
Caineng Cao, $\mathrm{MD}^{1,23}$ \\ Jingwei Luo, MD \\ Li Gao, MD' \\ Junlin Yi, MD ${ }^{1}$ \\ Xiaodong Huang, MD \\ Suyan Li, MD \\ Jianping Xiao, MD \\ Zhong Zhang, MD ${ }^{1}$ \\ Guozhen Xu, MD ${ }^{1}$
}

\section{${ }^{1}$ Department of Radiation Oncology, Cancer Hospital, Chinese Academy of Medical Sciences, Peking Union Medical College, Beijing, ${ }^{2}$ Department of Radiation Oncology, Zhejiang Cancer Hospital, Hangzhou, ${ }^{3}$ Zhejiang Key Laboratory of Radiation Oncology, Hangzhou, China}

Correspondence: Jingwei Luo, MD Department of Radiation Oncology, Cancer Hospital, Chinese Academy of Medical Sciences, Peking Union Medical College, No. 17, Panjiayuannanli, Chaoyang District, Beijing 100021, China

Tel: 86-10-87788282

Fax: 86-10-67706153

E-mail: jingweiluo2013@163.com

Received July 9, 2016

Accepted August 15, 2016

Published Online August 24, 2016

\begin{abstract}
Purpose
This study was conducted is to identify the prognostic value and staging categories of magnetic resonance imaging (MRI)-detected intracranial extension in nasopharyngeal carcinoma (NPC) with intensity-modulated radiotherapy (IMRT) to determine whether it is necessary to subclassify the T4 classification NPC.
\end{abstract}

\section{Materials and Methods}

A total of 335 nonmetastatic T4 classification NPC patients with MRI treated between March 2004 and June 2011 by radical IMRT were included. The T4 classification patients were subclassified into two grades (T4a, without intracranial extension vs. T4b, with intracranial extension) according to the site of invasion.

\section{Results}

The frequency of intracranial extension was $40.9 \%$ (137 of 335 patients). Multivariate analysis identified subclassification (T4a vs. T4b) as an independent prognostic factor for local failure-free survival ( $p=0.049$; hazard ratio [HR], 0.498) and overall survival ( $p=0.004 ; H R$, 0.572 ); however, it had no effect on regional failure-free survival or distant failure-free survival $(p>0.050)$.

\section{Conclusion}

For patients with T4 classification NPC, those with MRI-detected intracranial extension are more likely to experience local failure and death after IMRT than patients without intracranial extension. According to the site of invasion, subclassification of T4 patients as T4a or T4b has prognostic value in NPC.

\section{Introduction}

Nasopharyngeal carcinoma (NPC) remains endemic in southern China, with a peak annual incidence approaching 30 per 100,000 persons [1]. The primary purpose of the TNM classification system for NPC is to help physicians estimate the prognosis and design a management plan [2,3]. According to the seventh edition of the American Joint Committee on Cancer (AJCC) staging system, NPC with intracranial

\section{Key words}

Prognosis, Classification, Magnetic resonance imaging,

Nasopharyngeal carcinoma, Neoplasm staging extension and/or involvement of cranial nerves, hypopharynx, orbit, or extension to the infratemporal fossa/masticator space are classified as T4 [3]. This classification is primarily based on retrospective studies in which patients received conventional two-dimensional radiotherapy (2DRT) and computed tomography (CT) was the predominant pretreatment workup for tumor evaluation $[2,4]$. However, it has now become common practice to use magnetic resonance imaging (MRI) and intensity-modulated radiotherapy (IMRT) for imaging and treatment of NPC [4]. With these 
recent advances in both diagnostic and radiotherapeutic technologies, the 5-year overall survival (OS) rate for stage III-IV NPC has increased from $60 \%$ in the 2DRT era to $79 \%$ in the IMRT era [5].

NPC with intracranial extension may have inadequate radiation field coverage and dose owing to the proximity of the tumor to critical neural structures and the tolerance of critical organs such as the brain stem, temporal lobe, optic nerves, and chiasm. In our previous study [6], 70 patients with T4 classification NPC treated by IMRT were reported and 16 had developed local failures. The location and extent of locoregional failures were transferred to the pretreatment planning CT for dosimetry analysis. Of the 16 patients who developed local failures, seven were marginal, occurring in a steep dose gradient region at the margin of the high-dose planning target volume (PTV). Among the seven patients with marginal failures, six had intracranial extension on presentation, while one had involvement of cranial nerves and bone structures of the skull base. We concluded that the sites of marginal failure mainly occurred intracranially because of suboptimal dose coverage at the region proximal to the brain stem and temporal lobes. This study was conducted to evaluate the prognostic value of MRI-detected intracranial extension in NPC with IMRT, and to subclassify patients with T4 classification NPC according to the seventh edition of the AJCC staging system.

\section{Materials and Methods}

\section{Patient selection}

A total of 335 nonmetastatic T4 classification NPC patients treated by radical IMRT between March 2004 and June 2011 were included in this study. The pretreatment workup included a complete history and physical examination, liver and renal biochemical analysis, complete blood cell count, chest X-ray, fiber-optic nasopharyngoscopy, MRI of the head and neck, bone scintigraphy, ultrasonography of the abdominal region, and dental check. In addition, thoracic CT scan was required for $\mathrm{N} 3$ classification patients. All patients underwent disease staging using the AJCC 2010 staging system. The frequency of intracranial extension was 40.9\% (137 of 335 patients). The $\mathrm{T} 4$ classification patients were subclassified into two groups (T4a, without intracranial extension vs. T4b, with intracranial extension) according to the site of invasion. The T4a group included involvement of masticator space alone in 176, involvement of orbit alone in one, involvement of cranial nerves (assessed by neurological evaluation) alone in one, involvement of masticator space and orbit in six, involvement of masticator space and cranial nerves in 13, and involvement of masticator space and hypopharynx in one. The clinical characteristics are listed in Table 1.

\section{Magnetic resonance imaging}

All patients underwent MRI on a 1.5-T system (Signa Excite HD, General Electric, Milwaukee, WI) with an 8 channel head and neck phase array coil. The scan range covered the suprasellar cistern to the inferior margin at the sternal end of the clavicle. Non-enhanced series included axial T1-weighted imaging (fast spin echo [FSE], repetition time [TR]/echo time $[T E]=460 \mathrm{msec} / 8.3 \mathrm{msec}$ ), sagittal T1WI (FSE, TR/TE=500 msec/9.0 msec), axial T2WI (FSE with fatsuppressed, TR/TE=6,200 msec/92.9 msec). A contrastenhanced scan was performed using FSE with fat-suppressed after injection of gadopentetate dimeglumine (Magnevist, Schering, Berlin, Germany) with a dose of $0.2 \mathrm{~mL} / \mathrm{kg}$ and rate of $2.0 \mathrm{~mL} / \mathrm{sec}$ (TR/TE=295 msec/ $2.9 \mathrm{msec}$ for axial plane, TR/TE $=460 \mathrm{msec} / 7.8 \mathrm{msec}$ for sagittal and coronal plane). The thickness / slice gap were $5 \mathrm{~mm} / 1 \mathrm{~mm}$ for the axial plane and $5 \mathrm{~mm} / 0.5 \mathrm{~mm}$ for the sagittal and coronal plane, respectively. Two radiologists with more than 10 years of experience in MRI of head and neck cancers independently evaluated all scans, and any disagreements were resolved by consensus. In addition, a multidisciplinary team of head neck cancers in our center confirmed the extent of diseases and the treatment of patients with NPC.

The criterion for MRI involvement of the cavernous sinus was a change in contour or enlargement of the sinus. Skull base involvement included foramen ovale (OF) involvement only, foramen lacerum (FL) involvement only, both OF and FL involvement, skull base erosion, and involvement via the sphenoid sinus. Skull base erosion was diagnosed when destruction was present, even in the presence of OF and FL involvement [7].

\section{Treatment}

The techniques of planning and delivery of IMRT were described previously $[6,8]$. Additionally, we previously $[6,8]$ reported the patterns of failure, long-term survival and toxicity of T4 classification NPC after IMRT. Briefly, the dose prescribed was 70-76 Gy, 70 Gy, 60 Gy, and 50-56 Gy delivered within 6.5 weeks to the periphery of the gross tumor volume of the primary (GTVnx), nodal gross tumor volume (GTVnd), planning target volume (PTV1) of clinical target volume (CTV1) (i.e., high-risk regions), and PTV2 of CTV2 (i.e., low-risk regions), respectively, using the simultaneous integrated boost technique. The total dose of the GTVnx, GTVnd and PTV1 was given in 33 fractions. The total dose 
Table 1. Clinical characteristics

\begin{tabular}{|c|c|c|c|}
\hline \multirow{2}{*}{ Characteristic } & \multicolumn{3}{|c|}{ Total $(n=335)$} \\
\hline & T4a group (n=198) & T4b group $(n=137)$ & p-value \\
\hline \multicolumn{4}{|l|}{ Sex } \\
\hline Male & 139 & 113 & 0.010 \\
\hline Female & 59 & 24 & \\
\hline \multicolumn{4}{|l|}{ Age (yr) } \\
\hline Median & 45 & 47 & 0.289 \\
\hline Range & $12-77$ & $9-76$ & \\
\hline \multicolumn{4}{|l|}{ Pathology classification } \\
\hline Keratinizing & 1 & 1 & $>0.050$ \\
\hline Non-keratinizing & 197 & 136 & \\
\hline Involvement of masticator space & 196 & 116 & $<0.001$ \\
\hline Involvement of cranial nerves & 14 & 79 & $<0.001$ \\
\hline Involvement of orbit & 7 & 22 & $<0.001$ \\
\hline Involvement of hypopharynx & 1 & 0 & 0.405 \\
\hline \multicolumn{4}{|l|}{$\mathbf{N}$ classification } \\
\hline N0 & 15 & 11 & 0.593 \\
\hline N1 & 55 & 45 & \\
\hline N2 & 109 & 74 & \\
\hline N3a & 7 & 3 & \\
\hline $\mathrm{N} 3 \mathrm{~b}$ & 12 & 4 & \\
\hline \multicolumn{4}{|l|}{ Metastasis to retropharyngeal lymph nodes } \\
\hline Yes & 160 & 108 & 0.657 \\
\hline No & 38 & 29 & \\
\hline \multicolumn{4}{|l|}{ Boost irradiation } \\
\hline IMRT & 25 & 27 & 0.242 \\
\hline SBRT & 15 & 9 & \\
\hline \multicolumn{4}{|l|}{ Chemotherapy } \\
\hline Neoadjuvant & 8 & 6 & 0.221 \\
\hline Neoadjuvant plus concurrent & 9 & 8 & \\
\hline Concurrent & 132 & 78 & \\
\hline Concurrent plus adjuvant & 2 & 8 & \\
\hline Adjuvant & 3 & 3 & \\
\hline Neoadjuvant plus concurrent plus adjuvant & 1 & 1 & \\
\hline Neoadjuvant plus adjuvant & 4 & 1 & \\
\hline Radiotherapy alone & 39 & 24 & \\
\hline \multicolumn{4}{|l|}{ Targeted therapy } \\
\hline Cetuximab & 10 & 8 & 0.682 \\
\hline Nimotuzumab & 42 & 27 & \\
\hline
\end{tabular}

IMRT, intensity-modulated radiotherapy; SBRT, stereotactic body radiotherapy.

of the PTV2 was given in 28-30 fractions at 1.82-1.87 Gy per daily fraction. Two separate plans were made to accomplish the protocol.

Overall, 38 patients received neoadjuvant cisplatin-based chemotherapy, 239 received concurrent chemotherapy (cisplatin, $30 \mathrm{mg} / \mathrm{m}^{2} / \mathrm{wk}$ or $80 \mathrm{mg} / \mathrm{m}^{2} / 3 \mathrm{wk}$ ) and 23 received adjuvant cisplatin-based chemotherapy. Although chemotherapy was required for the treatment of locally advanced
NPC, the use of concurrent or adjuvant chemotherapy was not protocolized and it was used at the discretion of the attending physician of individual patients. Eighty-seven patients received cetuximab or nimotuzumab [9]. The patients did not receive concurrent chemotherapy because of advanced age, heart disease, hepatitis, severe diabetes, inadequate renal function, or patient refusal. Whenever possible, salvage treatments including surgery, reirradiation, 
and chemotherapy were administered to patients with persistent diseases or relapse.

\section{Treatment monitoring}

All patients were evaluated weekly during radiotherapy, and were required to be followed up upon completion of radiotherapy, 1 month after completion of radiotherapy, every 3 months during the first 2 years after completion, every 6 months from year 3 to year 5 , and annually thereafter. Each follow-up appointment consisted of a complete examination that included flexible fiberoptic endoscopy, abdomen ultrasound, chest X-ray, and basic serum chemistry. MRI of the head and neck was performed after the completion of IMRT and every 6 months thereafter.

\section{Statistical analysis}

The SPSS ver. 17.0 (SPSS Inc., Chicago, IL) was used for statistical analysis. The records of all patients were reviewed to assess the site or sites of treatment failures. Failure patterns were classified as local, regional, or distant. Category was determined at the time of the last follow-up. Local failure referred to failure of the primary tumor, while regional failure was defined as recurrence in regional lymph nodes and distant failure was defined as the appearance of tumor at a site representing hematogenous dissemination. The local failure-free survival (LFFS), regional failure-free survival (RFFS), distant failure-free survival (DFFS), and OS were estimated by use of the Kaplan-Meier method. LFFS, RFFS, DFFS, and OS were measured from day 1 of radiotherapy to the date of the event. The log-rank test was used in univariate analysis to assess differences between the T4a group and $\mathrm{T} 4 \mathrm{~b}$ group. Multivariate analyses with the Cox proportional hazards model were used to test independent significance by using backward elimination of insignificant explanatory variables. Host factors (age and sex) were included as covariates in all tests. Chi-squared, Fisher exact, and Student's $t$ tests were used to compare the differences between the T4a group and T4b group. All statistical analyses were two sided, and a $\mathrm{p}<0.05$ was considered to be statistically significant.

\section{Results}

\section{Outcome of the T4a group}

The median follow-up time for the T4a group was 53.2 months (range, 2.8 to 104.1 months). A total of 58 patients $(29.3 \%)$ developed treatment failures, with seven, two, and 37 developing local failure, regional failure, and distant failure, respectively, five developing locoregional failure, two developing distant failure and local failure, two developing distant failure and regional failure, and three developing distant failure and locoregional failure. In addition, one patient had treatment failure in the unilateral spared parotid gland. The 1-, 3-, and 5-year survival rates were as follows: OS, $95.9 \%, 83.1 \%$, and $71.5 \%$; LFFS, $98.4 \%, 92.0 \%$, and $90.3 \%$; RFFS, $98.4 \%, 93.2 \%$, and $93.2 \%$; DFFS, $88.1 \%, 80.3 \%$, and $75.9 \%$.
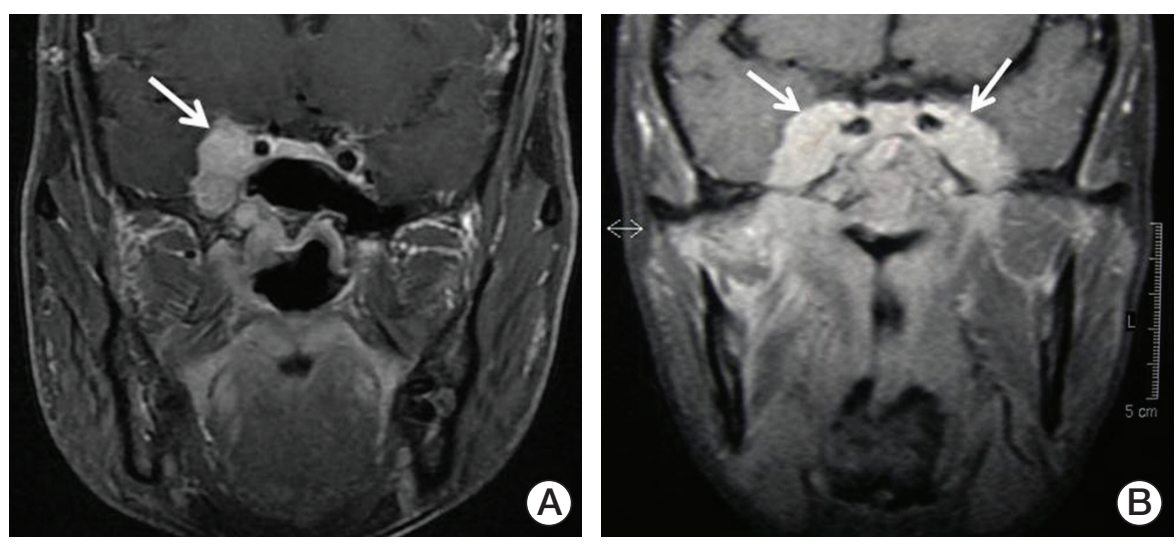

Fig. 1. Representative magnetic resonance image of two nasopharyngeal carcinoma patients with intracranial extension. (A) Coronal T1-weighted fat saturation postcontrast enhanced image of limited intracranial involvement (arrow). (B) Coronal T1-weighted fat saturation postcontrast enhanced image of extensive intracranial involvement (arrows). 
A
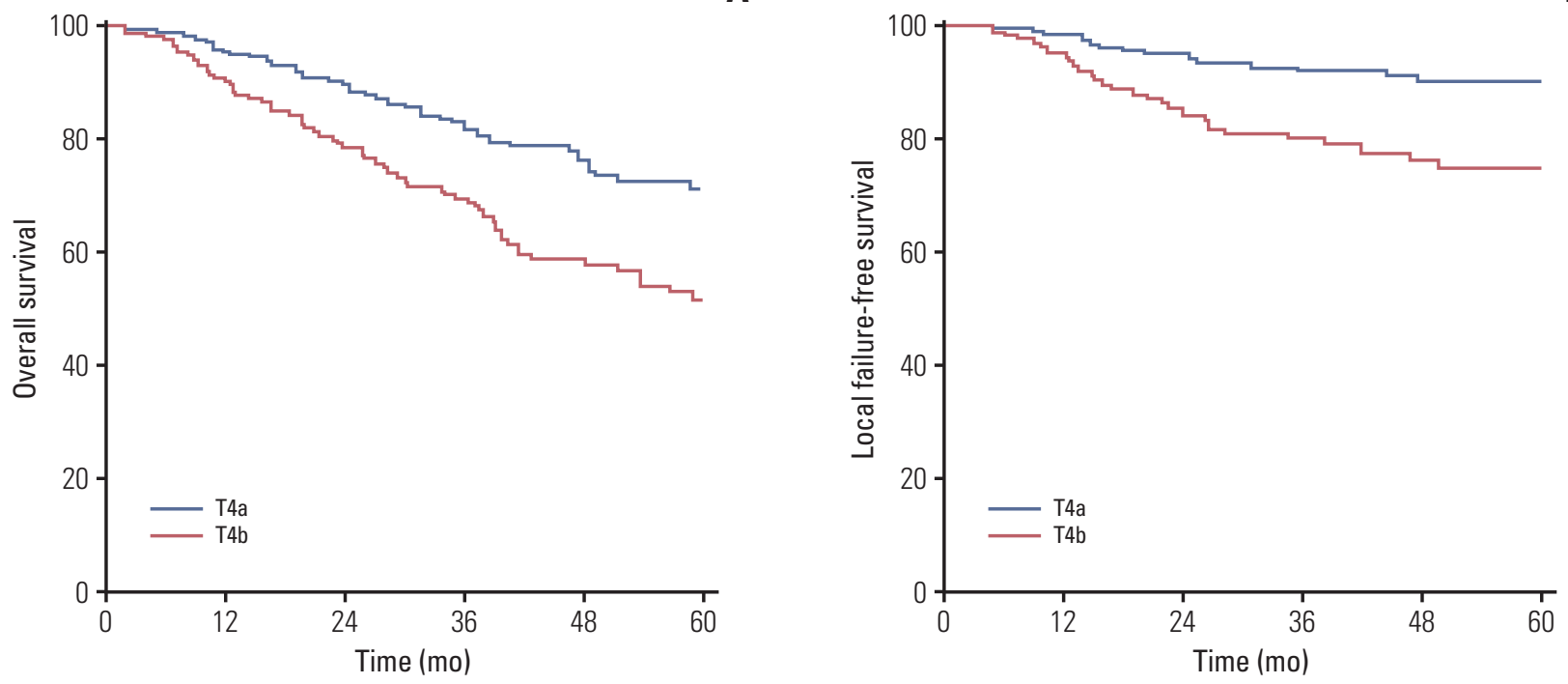

C
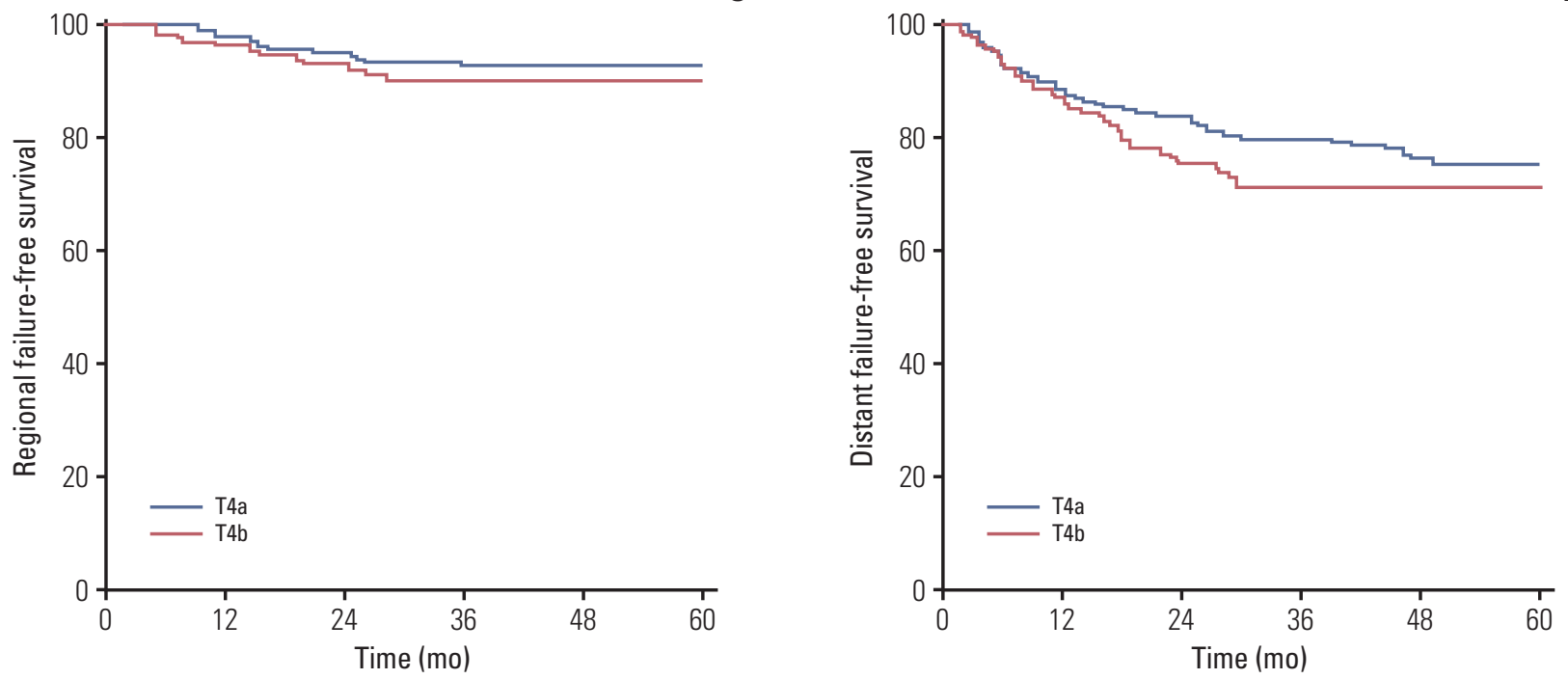

Fig. 2. Kaplan-Meier curve showing overall survival (A), local failure-free survival (B), regional failure-free survival (C), and distant failure-free survival (D) of the T4a group and the T4b group in the study.

\section{Outcome of the $T 4 b$ group}

The median follow-up time for the T4b group was 56.7 months (range, 10.8 to 114.9 months). A total of 60 patients (43.8\%) developed treatment failure, among which 17 , one, and 26 developed local failure, regional failure, and distant failure, respectively, three developed locoregional failure, six developed distant failure and local failure, five developed distant failure and regional failure, and two developed dis- tant failure and locoregional failure. In addition, two of these patients experienced treatment failure in the unilateral spared parotid gland. The 1-, 3-, and 5-year survival rates were as follows: OS, $91.2 \%, 69.7 \%$, and $51.6 \%$; LFFS, 95.4\%, $79.9 \%$, and $74.8 \%$; RFFS, $96.9 \%, 90.7 \%$, and $90.7 \%$; DFFS, $86.6 \%, 71.6 \%$, and $71.6 \%$.

All T4b patients were subclassified according to the extent of intracranial involvement as follows: limited involvement was defined as a primary nasopharyngeal tumor involving 
Table 2. Multivariate analysis of prognostic factors for patients with nasopharyngeal carcinoma

\begin{tabular}{llccc} 
Endpoint & Item & HR & $95 \%$ CI & p-value \\
\hline LFFS & N classification (N2-3 vs. N0-1) & 1.941 & $0.995-3.788$ & 0.052 \\
& Subclassification (T4a vs. T4b) & 0.498 & $0.248-0.997$ & 0.049 \\
& Boost (no vs. yes) & 0.507 & $0.270-0.951$ & 0.034 \\
RFFS & N classification (N2-3 vs. N0-1) & 6.897 & $1.609-29.559$ & 0.009 \\
DFFS & N classification (N2-3 vs. N0-1) & 2.028 & $1.206-3.410$ & 0.008 \\
OS & Age & 0.440 & $0.300-0.644$ & $<0.001$ \\
& N classification (N2-3 vs. N0-1) & 1.690 & $1.116-2.557$ & 0.013 \\
& Boost (no vs. yes) & 0.652 & $0.429-0.992$ & 0.046 \\
& Subclassification (T4a vs. T4b) & 0.572 & $0.389-0.839$ & 0.004 \\
\hline
\end{tabular}

HR, hazard ratio; CI, confidence interval; LFFS, local failure-free survival; RFFS, regional failure-free survival; DFFS, distant failure-free survival; OS, overall survival.

the unilateral cavernous sinus or parasellar region only; extensive involvement was defined as extension of the tumor to the bilateral cavernous sinus or the parasellar region to the prepontine region or the posterior cranial fossa [10]. Of these patients, 90 patients were classified as having limited involvement (Fig. 1A) and 47 as having extensive involvement (Fig. 1B). Univariate analysis showed no significant differences in terms of OS (5-year, 53.4\% vs. $47.0 \%$; $\mathrm{p}=0.927$ ), LFFS (5-year, 74.2\% vs. 76.2\%; $\mathrm{p}=0.830$ ), RFFS (5-year, $90.8 \%$ vs. $90.1 \% ; \mathrm{p}=0.929$ ), and DFFS (5-year, $72.9 \%$ vs. $69.2 \%$; $\mathrm{p}=0.780$ ) between patients with limited involvement and extensive involvement.

\section{Outcome of the whole group}

The median follow-up time for the whole group was 53.6 months (range, 2.8 to 114.9 months). For all patients, the 1-, 3 -, and 5-year LFFS rates were $97.2 \%, 87.2 \%$, and $84.1 \%$; RFFS rates were $97.8 \%, 92.2 \%$, and $92.2 \%$; DFFS rates were $87.4 \%$, $76.8 \%$, and $74.1 \%$; and OS rates were $93.9 \%, 77.5 \%$, and $63.0 \%$, respectively. The 5-year LFFS rate and the 5-year OS rate in the T4a group were higher than for patients in the $\mathrm{T} 4 \mathrm{~b}$ group ( $\mathrm{p}=0.001$ and $\mathrm{p}=0.001$ ). No significant differences was observed in RFFS and DFFS between the T4a group and the T4b group ( $\mathrm{p}=0.405$ and $\mathrm{p}=0.155$ ) (Fig. 2 ).

Multivariate analysis was performed to adjust for various prognostic factors, and the following parameters were included in the Cox proportional hazards model: age, sex, $\mathrm{N}$ classification (N2-3 vs. N0-1), metastasis to retropharyngeal lymph nodes, boost irradiation, volume of GTVnx ( $>50$ $\mathrm{mL}$ vs. $\leq 50 \mathrm{~mL}$ ), concurrent chemotherapy, and subclassification of T4 patients (T4a vs. T4b). Subclassification of T4 was identified as an independent prognostic factor for both OS (hazard ratio [HR], 0.572; 95\% confidence interval [CI], 0.389 to $0.839 ; \mathrm{p}=0.004$ ) and LFFS (HR, $0.498 ; 95 \% \mathrm{CI}, 0.248$ to 0.997 ; $\mathrm{p}=0.049$ ) with use of multivariate analysis. $\mathrm{N}$ classification was found to be an independent prognostic factor for both RFFS and DFFS. The outcomes of multivariate analysis are shown in Table 2.

\section{Discussion}

Our study indicated that subclassification of T4 patients (T4a vs. T4b) was an independent prognostic factor of OS and LFFS for patients with T4 classification NPC treated by radical IMRT. In 2001, four criteria were defined for a good staging classification system: (1) survival rates among the groups (hazard discrimination); (2) given group including the subgroups defined by $\mathrm{T}, \mathrm{N}$, and $\mathrm{M}$ within a grouping system having similar survival rates (hazard consistency); (3) balanced distribution of patients among groups; and (4) high prediction of cure (outcome prediction) [11]. Therefore, we recommend that the subclassification of T4 patients (T4a vs. T4b) be used for NPC in the TNM classification system.

Compared with 2DRT, IMRT could achieve very good local control for NPC [12-14]. Sun et al. [12] reported that LFFS were significantly different among groups of patients with different $\mathrm{T}$ classifications except groups of patients with $\mathrm{T} 1$ and $\mathrm{T} 2$ classification, whereas two other studies indicated that $\mathrm{T}$ classification was not an independent prognostic factor for local control $[13,14]$. In a study [15] of head and neck squamous cell carcinoma treated by radical radiotherapy, patients receiving higher doses ( $\geq 66 \mathrm{~Gy}$ ) had better outcomes than those that received lower doses. For NPC treated by IMRT, administering radiation at above $66.5 \mathrm{~Gy}$ to the target yielded significantly less local failure [14]. Owing to the proximity of the tumor to critical neural structures and the 
tolerance of critical organs such as the brain stem and temporal lobe, NPC with intracranial extension may not have adequate radiation field coverage and dose, even in the era of IMRT. Hu et al. [10] reported that limited intracranial involvement was associated with better OS and locoregional recurrence-free survival in T4 classification NPC. However, in our study, univariate analysis revealed no significant differences in terms of OS, LFFS, RFFS, and DFFS between patients with limited involvement and extensive involvement, which was similar to the results of NPC treated by IMRT using MRI for pretreatment workup [2]. This was likely because all of the patients in the study conducted by $\mathrm{Hu}$ et al. [10] received 2DRT, some patients underwent CT for pretreatment workup, and only 31 patients (39.1\%) received concurrent chemoradiotherapy.

To the best of our knowledge, NPC with intracranial extension always invades through the skull base and neural foramina. Intracranial extension is frequently associated with MRI-detected intracranial nerve involvement, and MRIdetected intracranial nerve involvement is an independent prognostic factor of both OS and metastasis-free survival for NPC [16]. Moreover, invasion into the marrow of the skull base bones is associated with distant metastasis in patients with NPC [17]. Hung et al. [18] reported that prepontine cistern invasion was an independent prognostic factor of distant metastasis-free survival for T4 classification NPC treated by IMRT. Chen et al. [4] reported that involvement of the masticator space alone was positively associated with OS and distant metastasis-free survival for T4 classification NPC. More patients with involvement of cranial nerves were included in the T4b group $(\mathrm{p}<0.001)$, while 176 patients $(88.9 \%)$ with involvement of the masticator space alone were included in the T4a group. Although no significant difference in DFFS was observed between the T4a group and the T4b group, the 5-year DFFS rate in the T4a group was higher than that for patients in the T4b group (75.9\% vs. $71.6 \%)$.

Strengthening treatment may be immediately administered to NPC with intracranial extension. Although concurrent chemoradiotherapy is the standard treatment for locoregionally advanced NPC, the role of adjuvant chemotherapy is still debated [1,19-22]. Niu et al. [23] evaluated 32 patients with intracranial invasion NPC treated with neoadjuvant chemotherapy and replanning IMRT with concurrent chemotherapy in which the doses to the brain stem, optic nerve, optic chiasm, and temporal lobe were reduced and the 2-year local control rates and DFFS were $88.2 \%$ and $89.6 \%$, respectively. Neoadjuvant chemotherapy and new chemotherapy regimens should be evaluated for NPC with intracranial extension in clinical trials.

It should be noted that the present study had several limitations. Specifically, different systemic treatments (i.e., neoadjuvant chemotherapy vs. concurrent chemotherapy vs. adjuvant chemotherapy) were used. Concurrent chemoradiotherapy is currently considered the standard of care for non-metastatic T4 classification NPC [1,19-22]. In the present study, 239 patients $(71.3 \%)$ received concurrent chemotherapy, and only patients who received radical IMRT were included, which could have affected the outcomes. Furthermore, the use of 6/5.5-mm-thick sections for MRI in the present study could have resulted in the omission of some information. Therefore, it would be desirable to use thinner sections (i.e., 3-mm-thick) for MRI in the future.

\section{Conclusion}

For patients with T4 classification NPC, those with MRIdetected intracranial extension were more likely to experience local failure and death after IMRT than patients without intracranial extension. According to the site of invasion, subclassification of $\mathrm{T} 4$ patients as $\mathrm{T} 4 \mathrm{a}$ or $\mathrm{T} 4 \mathrm{~b}$ has prognostic value in NPC.

\section{Conflicts of Interest}

Conflict of interest relevant to this article was not reported.

\section{Acknowledgments}

This retrospective study was approved by the independent ethics committee of the Cancer Hospital, Chinese Academy of Medical Sciences (No.15-117/1044). The requirement to obtain informed consent was waived. 


\section{References}

1. Lee AW, Ma BB, Ng WT, Chan AT. Management of nasopharyngeal carcinoma: current practice and future perspective. J Clin Oncol. 2015;33:3356-64.

2. Ng WT, Yuen KT, Au KH, Chan OS, Lee AW. Staging of nasopharyngeal carcinoma: the past, the present and the future. Oral Oncol. 2014;50:549-54.

3. Edge SB, Compton CC. The American Joint Committee on Cancer: the 7th edition of the AJCC cancer staging manual and the future of TNM. Ann Surg Oncol. 2010;17:1471-4.

4. Chen L, Liu LZ, Chen M, Li WF, Yin WJ, Lin AH, et al. Prognostic value of subclassification using MRI in the 4 classification nasopharyngeal carcinoma intensity-modulated radiotherapy treatment. Int J Radiat Oncol Biol Phys. 2012;84:196202.

5. Lee AW, Ng WT, Chan LL, Hung WM, Chan CC, Sze HC, et al. Evolution of treatment for nasopharyngeal cancer: success and setback in the intensity-modulated radiotherapy era. Radiother Oncol. 2014;110:377-84.

6. Cao CN, Luo JW, Gao L, Yi JL, Huang XD, Wang K, et al. Clinical outcomes and patterns of failure after intensity-modulated radiotherapy for T4 nasopharyngeal carcinoma. Oral Oncol. 2013;49:175-81.

7. Chong VF, Fan YF, Khoo JB. Nasopharyngeal carcinoma with intracranial spread: CT and MR characteristics. J Comput Assist Tomogr. 1996;20:563-9.

8. Cao CN, Luo JW, Gao L, Yi JL, Huang XD, Wang K, et al. Update report of $\mathrm{T} 4$ classification nasopharyngeal carcinoma after intensity-modulated radiotherapy: an analysis of survival and treatment toxicities. Oral Oncol. 2015;51:190-4.

9. Huang XD, Yi JL, Gao L, Xu GZ, Jin J, Yang WZ, et al. Multicenter phase II clinical trial of humanized anti-epidermal factor receptor monoclonal antibody h-R3 combined with radiotherapy for locoregionally advanced nasopharyngeal carcinoma. Zhonghua Zhong Liu Za Zhi. 2007;29:197-201.

10. Hu YC, Chang CH, Chen CH, Ger LP, Liu WS, Lin LC, et al. Impact of intracranial extension on survival in stage IV nasopharyngeal carcinoma: identification of a subset of patients with better prognosis. Jpn J Clin Oncol. 2011;41: 95-102.

11. Groome PA, Schulze K, Boysen M, Hall SF, Mackillop WJ. A comparison of published head and neck stage groupings in carcinomas of the oral cavity. Head Neck. 2001;23:613-24.

12. Sun X, Su S, Chen C, Han F, Zhao C, Xiao W, et al. Long-term outcomes of intensity-modulated radiotherapy for 868 patients with nasopharyngeal carcinoma: an analysis of survival and treatment toxicities. Radiother Oncol. 2014;110:
398-403.

13. Lin S, Pan J, Han L, Guo Q, Hu C, Zong J, et al. Update report of nasopharyngeal carcinoma treated with reduced-volume intensity-modulated radiation therapy and hypothesis of the optimal margin. Radiother Oncol. 2014;110:385-9.

14. Ng WT, Lee MC, Hung WM, Choi CW, Lee KC, Chan OS, et al. Clinical outcomes and patterns of failure after intensitymodulated radiotherapy for nasopharyngeal carcinoma. Int J Radiat Oncol Biol Phys. 2011;79:420-8.

15. Dinshaw KA, Agarwal JP, Ghosh-Laskar S, Gupta T, Shrivastava SK. Radical radiotherapy in head and neck squamous cell carcinoma: an analysis of prognostic and therapeutic factors. Clin Oncol (R Coll Radiol). 2006;18:383-9.

16. Liu L, Liang S, Li L, Mao Y, Tang L, Tian L, et al. Prognostic impact of magnetic resonance imaging-detected cranial nerve involvement in nasopharyngeal carcinoma. Cancer. 2009;115: 1995-2003.

17. Cheng SH, Tsai SY, Yen KL, Jian JJ, Feng AC, Chan KY, et al. Prognostic significance of parapharyngeal space venous plexus and marrow involvement: potential landmarks of dissemination for stage I-III nasopharyngeal carcinoma. Int J Radiat Oncol Biol Phys. 2005;61:456-65.

18. Hung TM, Chen CC, Lin CY, Ng SH, Kang CJ, Huang SF, et al. Prognostic value of prepontine cistern invasion in nasopharyngeal carcinoma treated by intensity-modulated radiotherapy. Oral Oncol. 2014;50:228-33.

19. Loong HH, Chan AT. Controversies in the systemic treatment of nasopharyngeal carcinoma. Oral Oncol. 2014;50:785-90.

20. Baujat B, Audry H, Bourhis J, Chan AT, Onat H, Chua DT, et al. Chemotherapy in locally advanced nasopharyngeal carcinoma: an individual patient data meta-analysis of eight randomized trials and 1753 patients. Int J Radiat Oncol Biol Phys. 2006;64:47-56.

21. Langendijk JA, Leemans CR, Buter J, Berkhof J, Slotman BJ. The additional value of chemotherapy to radiotherapy in locally advanced nasopharyngeal carcinoma: a meta-analysis of the published literature. J Clin Oncol. 2004;22:4604-12.

22. Blanchard P, Lee A, Marguet S, Leclercq J, Ng WT, Ma J, et al. Chemotherapy and radiotherapy in nasopharyngeal carcinoma: an update of the MAC-NPC meta-analysis. Lancet Oncol. 2015;16:645-55.

23. Niu X, Chang X, Gao Y, Hu C, Kong L. Using neoadjuvant chemotherapy and replanning intensity-modulated radiotherapy for nasopharyngeal carcinoma with intracranial invasion to protect critical normal tissue. Radiat Oncol. 2013;8:226. 